\title{
Tensão de Crescimento no Lenho de Eucalyptus benthamii e sua Relação com Características Dendrométricas em Diferentes Espaçamentos
}

\author{
Graziela Baptista Vidaurre ${ }^{1}$, Leif Nutto ${ }^{2}$, Frederico José Nistal França ${ }^{3}$, \\ Rafael Leite Braz ${ }^{4}$, Luciano Farinha Watzlawick ${ }^{5}$, Jordão Cabral Moulin ${ }^{3}$ \\ ${ }^{1}$ Departamento de Ciências Florestais e da Madeira, Universidade Federal do Espirito Santo - UFES, \\ Jerônimo Monteiro/ES, Brasil \\ ${ }^{2}$ Malinovski Florestal, Curitiba/PR, Brasil \\ ${ }^{3}$ Programa de Pós-graduação em Ciências Florestais, Universidade Federal do Espírito Santo - UFES, \\ Jerônimo Monteiro/ES, Brasil \\ ${ }^{4}$ Programa de Pós-graduação em Engenharia Florestal, Universidade Federal do Paraná - UFPR, \\ Curitiba/PR, Brasil \\ ${ }^{5}$ Unicentro, Guarapuava/PR, Brasil
}

\section{RESUMO}

O objetivo deste trabalho foi avaliar a influência do espaçamento do plantio da madeira de eucalipto, quanto às suas características dendrométricas e a deformação residual longitudinal (DRL) da madeira e as relações entre essas variáveis. O material utilizado foi o Eucalyptus benthamii aos 5 anos de idade nos espaçamentos $2 \times 3,3 \times 3,3 \times 4$ e $4 \times 4 \mathrm{~m}$, a projeção da copa foi determinada através da medição de oito raios com ângulos variáveis, sendo elaborados mapas da distribuição e projeção da copa. As medições da DRL foram à $1,30 \mathrm{~m}$ do solo nas quatro direções cardinais, em árvores vivas, com o auxílio de extensômetro (Growth Strain Gauge), pelo método CIRAD-Fôret. Nos resultados houve grande variabilidade das variáveis de crescimento e dos níveis de tensão de crescimento para os diferentes espaçamentos. Os menores valores médios de DRL foram observados na direção sul, o aumento do espaçamento não alterou significativamente os valores da DRL. As correlações de maiores magnitudes ocorreram entre os valores máximos de DRL e as variáveis de crescimento árvore.

Palavras-chave: deformação residual longitudinal, variáveis de crescimento, Eucalyptus.

\section{Growth Stress in Eucalyptus benthamii and its Relationship with Dendrometric Characteristics at Different Spacings}

\begin{abstract}
The aim of this study was to evaluate the influence of planting eucalyptus wood with respect to its dendrometric characteristics and longitudinal residual strain (LRS) of the wood, and the relationships between these variables. The material used was Eucalyptus benthamii at five years of age in $2 \times 3,3 \times 3,3 \times 4$ and $4 \times 4 \mathrm{~m}$ spacing. The projection of the crown was determined by measuring eight spokes with variable angles, and maps of crown distribution and projection were prepared. Measurements of LRS were taken $1.30 \mathrm{~m}$ above the ground in the four cardinal directions in living trees, with the aid of strain meters (Growth Strain Gauge) by the CIRAD-Forêt method. There was great variability in the results of the growth variables and levels of growth stresses
\end{abstract}


for different spacing. The lowest average values of LRS were observed in the south direction, and increased spacing did not significantly alter the LRS values. Correlations between LRS and growth variables were only found with maximum LRS values for each tree.

Keywords: longitudinal residual deformation, growth variables, Eucalyptus.

\section{INTRODUÇÃO}

Espécies do gênero Eucalyptus são amplamente utilizadas em plantios comerciais em diferentes regiões do mundo. Alzate et al. (2005) destacaram a sua capacidade produtiva, adaptabilidade a diversos ambientes e, sobretudo, a expressiva diversidade de espécies que possui, tornando possível atender os requisitos tecnológicos dos mais diversos segmentos da produção industrial madeireira.

O uso do eucalipto na indústria madeireira para produtos sólidos, tais como serraria e laminação, desde as primeiras tentativas causaram diversos problemas. Devido a altas tensões de crescimento, como frequentemente encontradas em espécies folhosas classificadas nos mercados como hardwood, a madeira roliça e os produtos da primeira conversão apresentam rachaduras, trincas e empenamentos (Archer, 1986; Yang \& Waugh, 2001). Para Ferrand (1983), as tensões de crescimento estão em equilíbrio enquanto as árvores estão de pé, posteriormente ao corte ocorrem imediatamente deformações e rachaduras nos topos de toras, em função da modificação do estado de equilíbrio que vigorava durante o crescimento.

As tensões de crescimento são medidas a partir da variação do comprimento de uma peça de madeira quando ocorre liberação das suas junções a outros elementos vizinhos, nessa mensuração é determinada a deformação residual longitudinal - DRL (Lisboa, 1993).

Existem na literatura estudos que relatam uma correlação entre a formação de tensões de crescimento e variáveis de crescimento, tais como o diâmetro na altura de 1,30 m do solo (DAP), a altura, tamanho e forma da copa das árvores (Vignote et al., 1996; Lemos, 2002; Nutto \& Touza Vázquez, 2006a). Cardoso et al. (2007) descreveram que diversas técnicas vêm sendo adotadas para melhorar a qualidade da madeira e seu rendimento, entre elas o uso de técnicas silviculturais como desrama, espaçamento dos plantios e idade, as quais apresentam grande influência na qualidade da madeira.
Conforme relatado por Kubler (1988), tratamentos de silvicultura como o desbaste podem minimizar tensões de crescimento ao manter uma taxa de crescimento constante e as condições de crescimento estáveis. Isso não é surpreendente, pois a formação de tensões está altamente vinculada às condições de crescimento da árvore individual (Archer, 1987; Kubler, 1987). Os processos fisiológicos e as forças físicas que influenciam na formação das tensões de crescimento estão descritas nos trabalhos de Kubler (1988) e Vidaurre et al. (2013): de acordo com esses autores, os níveis da tensão de crescimento são influenciados pelos manejos e tratos silviculturais.

A relação entre a dimensão da copa e o diâmetro na altura de $1,30 \mathrm{~m}$ (DAP) foi descrita por vários autores, também para diferentes espécies de eucalipto (Nutto \& Touza Vázquez, 2006a; Nutto et al., 2006; Medhurst et al., 1999). Para o Eucalyptus benthamii, no Brasil, ainda não existem conhecimentos sobre a relação do tamanho da copa com o crescimento em diâmetro e o possível impacto no nível das tensões de crescimento. Diante desse contexto, o presente trabalho teve como objetivo avaliar as variáveis de crescimento em diferentes espaçamentos e sua relação com a deformação residual longitudinal (DRL) decorrente das tensões de crescimento nas árvores de Eucalyptus benthamii.

\section{MATERIAL E MÉTODOS}

Os dados para este estudo foram coletados em plantios de Eucalyptus benthamii com 5 anos de idade, oriundos de semente, implantados sob os diferentes espaçamentos, sendo eles 2,0 $\times 3,0 \mathrm{~m} ; 3,0 \times 3,0 \mathrm{~m} \mathrm{e} 3,0 \times 4,0 \mathrm{~m} \mathrm{e} 4,0 \times 4,0 \mathrm{~m}$, localizados na área experimental do Departamento de Agronomia da Universidade Estadual Centro Oeste do Paraná, situada no município de Guarapuava, na região centro-sul do estado do Paraná.

A área experimental localiza-se a $25^{\circ} 23^{\prime} 36^{\prime \prime}$ de latitude sul e $51^{\circ} 27^{\prime} 19^{\prime \prime}$ 'de longitude oeste e a $1.120 \mathrm{~m}$ de altitude, apresentando o terreno uma pequena inclinação para o sudoeste $\left(2^{\circ}\right)$, com ventos predominantes na 
direção leste. O clima é classificado, segundo Köppen, como subtropical mesotérmico úmido ( $\mathrm{Cfb}$ ), sem estação seca, verões frescos e inverno moderado. Apresenta uma temperatura média do mês mais quente inferior a $22{ }^{\circ} \mathrm{C}$ e a precipitação média anual é de $2.030 \mathrm{~mm}$ (Godoy et al., 1976).

Como variáveis de crescimento consideraram-se o diâmetro a 1,30 m de altura do solo (DAP), a altura total da árvore, da inserção da copa e a projeção da copa (altura e diâmetro). O diâmetro na altura do peito foi mensurado através de uma fita diamétrica, já a altura total e a altura de inserção da copa (definida no primeiro galho vivo) foram tomadas com o aparelho denominado hipsômetro Vertex IV.

A projeção da copa foi determinada através da medição de oito raios com ângulos variáveis de acordo com Nutto \& Spiecker (2000) e, como pode ser observado na Figura 1, foram elaborados mapas da distribuição e projeção da copa. Segundo Röhle (1986), esse método resulta em melhor precisão e efetividade dos resultados com árvores de copas simétricas e assimétricas. Os raios foram medidos com o auxílio de uma fita métrica e uma bússola, também foi utilizado um clinômetro de Suunto para projetar os pontos que melhor representavam a forma real da projeção da copa no solo. Em seguida, tais pontos foram determinados pela distância e o azimute da árvore, de acordo com Spiecker (1983).

Dos polígonos obtidos na projeção da copa foi calculada, de acordo com Röhle (1986), a área da copa das árvores através do método triangular. Em seguida, as áreas dos polígonos foram transformadas em círculos e calculou-se também a largura média da copa. Foi gerado um croqui com um script incluído no ArcGis 3.2 para visualização e a análise do desenvolvimento das copas em cada parcela (Figura 2).

Com os dados obtidos também foram calculados o quociente entre altura e diâmetro (altura - $\mathrm{m}$ - dividida pelo diâmetro - cm) e a excentricidade da copa, que é a distância do ponto de equilíbrio do polígono da copa com o centro do fuste em 1,3 m de altura. Finalmente foi calculado o diâmetro da copa, através da área dos polígonos da copa medidos, assumindo que essa área é um círculo. Esse valor também foi usado para a comparação com o DAP, para se ter valores da mesma dimensão (distâncias).

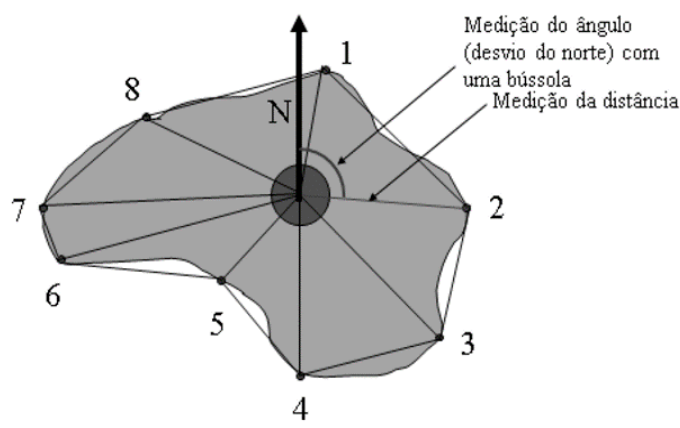

Figura 1. Demonstração da medição da copa com oito raios e ângulos variáveis das árvores de Eucalyptus benthamii.

Figure 1. Demonstration measuring cup with eight spokes and variable angles Eucalyptus benthamii trees.

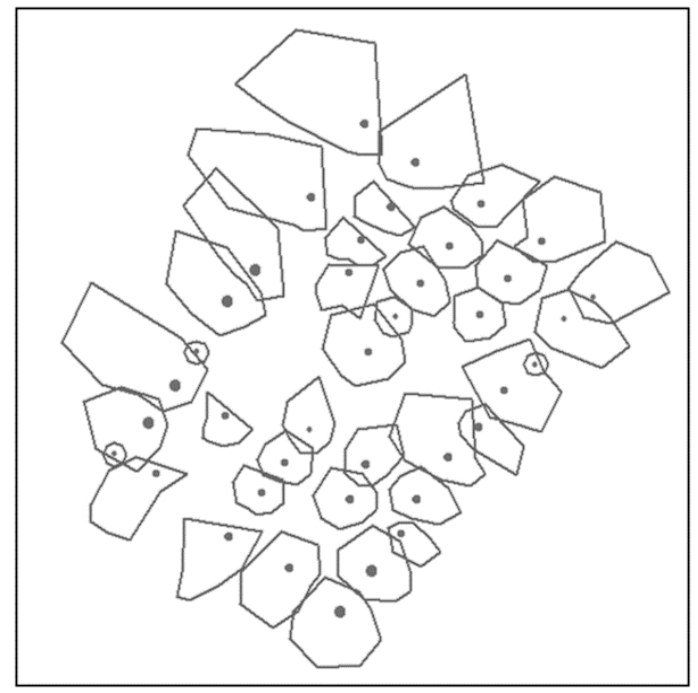

Figura 2. Croqui da distribuição e desenvolvimento das copas das árvores de Eucalyptus benthamii (projeção bidimensional).

Figure 2. Sketch the distribution and development of the trees of Eucalyptus benthamii (bi-dimensional projection).

As medições das deformações residuais longitudinais (DRL) foram realizadas de acordo com o método não destrutivo do CIRAD-Forêt, através do extensômetro Growth Strain Gauge - medidor de deformação de crescimento, que consiste em um relógio comparador digital que tem como função informar a deformação residual longitudinal por meio da compressão das fibras, uma vez que a tensão é liberada. Após a remoção da casca das árvores na altura do DAP, o extensômetro foi apoiado em dois pinos fixados no tronco, com uma distância entre eles de $45 \mathrm{~mm}$ na direção da grã. 
Posteriormente foi feito um furo de $20 \mathrm{~mm}$ de diâmetro com um arco de pua, entre os pinos, a fim de liberar as tensões refletidas na contração longitudinal das fibras, obtendo-se assim a deformação residual longitudinal da madeira. Ao romper os tecidos do lenho é liberada a tensão e o valor em milímetros é registrado diretamente no visor do relógio digital.

As medições referentes à deformação residual longitudinal decorrentes dos níveis de tensões de crescimento foram tomadas ao redor do tronco na altura de 1,30 m do solo nas diferentes direções cardeais, norte, sul, leste e oeste. Em cada bloco foram selecionadas cinco árvores representativas. A representatividade foi avaliada através da medição do diâmetro na altura do peito (DAP) de todas as árvores do bloco, como representativas foram escolhidos aqueles indivíduos que estavam com diâmetro médio +/- um desvio padrão. Para cada espaçamento, um bloco com uma repetição foram escolhidos, medindo-se assim 40 árvores dentro dos blocos experimentais. Para avaliar a relação do DAP e a projeção da copa foram selecionadas e mensuradas cinco árvores adicionais com os maiores diâmetros encontrados. Essas foram localizadas nas bordaduras do experimento. Árvores de bordadura geralmente sofrem menos competição, desenvolvem copas maiores e assim mostram um crescimento em diâmetro superior àquelas dentro dos povoamentos (Nutto \& Touza Vázquez, 2006b).

Para se avaliar o grau de significância dos parâmetros avaliados, bem como suas variações, foi realizada uma análise de variância e, quando significativa, aplicou-se o teste Tukey a 5\% de probabilidade para a comparação de médias. Posteriormente foi conduzido um estudo das correlações entre as variáveis de crescimento e a deformação residual longitudinal.

\section{RESULTADOS E DISCUSSÃO}

Os valores médios para as variáveis de crescimento DAP, altura total e altura de inserção da copa para os diferentes espaçamentos encontram-se na Tabela 1. Em relação ao DAP, observou-se um comportamento já esperado, ou seja, quanto maior o espaçamento de plantio, maior foi o DAP, o que resultou em acréscimos de área basal e, consequentemente, aumentos volumétricos. Os dois menores espaçamentos de plantio não diferiram significativamente pelo teste de Tukey a $5 \%$ com relação ao DAP, ao passo que os espaçamentos $3,0 \times 4,0 \mathrm{~m} \mathrm{e}$ $4,0 \times 4,0 \mathrm{~m}$ apresentaram diferenças. Isso se deve a concorrência pelo dossel, que é maior em menores espaços disponíveis. Cardoso (1989) ressaltou que povoamentos muito densos tendem naturalmente a apresentar árvores de diâmetro menor se comparados com árvores de povoamentos pouco densos, onde a concorrência por espaço é menor e o crescimento em diâmetro é mais acentuado.

Apesar de ter ocorrido diferença entre espaçamentos para altura de árvores, essa característica não seguiu um padrão definido. Segundo Evert (1971), existem casos onde a altura média aumenta com o espaçamento e outros onde o resultado é o inverso.

O maior espaçamento de plantio $(4,0 \times 4,0 \mathrm{~m})$ diferiu significativamente dos demais espaçamentos quanto à inserção de primeiro galho. A altura de inserção do primeiro galho sofreu um efeito maior nos diferentes espaçamentos se comparada à altura total das árvores. É possível perceber que com o aumento do espaçamento a altura de inserção do primeiro galho reduziu, isso se deve ao maior espaço disponível para desenvolvimento de galhos laterais. Através da variável inserção da copa em combinação com a altura total, determina-se o comprimento da copa, uma variável frequentemente

Tabela 1. Valores médios e coeficiente de variação das variáveis de crescimento para os diferentes espaçamentos em plantios de Eucalyptus benthamii aos 5 anos de idade.

Table 1. Mean values and coefficient of variation of the growth parameters for different spacings in Eucalyptus benthamii to 5 years old.

\begin{tabular}{clll} 
Espaçamento $(\mathbf{m} \times \mathbf{m})$ & \multicolumn{1}{c}{ DAP $(\mathbf{c m})$} & Ht $(\mathbf{m})$ & Hic $(\mathbf{m})$ \\
\hline $\mathbf{2 , 0} \times \mathbf{3 , 0}$ & $\mathbf{1 6 , 9 8} \mathrm{a}^{*}(7,64)^{\star *}$ & $\mathbf{2 2 , 7 1} \mathrm{a}(9,29)$ & $\mathbf{1 5 , 7 3} \mathrm{a}(15,75)$ \\
$\mathbf{3 , 0} \times \mathbf{3 , 0}$ & $\mathbf{1 7 , 9 8} \mathrm{a}(4,36)$ & $\mathbf{2 4 , 6 4} \mathrm{b}(3,03)$ & $\mathbf{1 6 , 8 6} \mathrm{a}(5,99)$ \\
$\mathbf{3 , 0} \times \mathbf{4 , 0}$ & $\mathbf{2 0 , 2 0} \mathrm{b}(10,81)$ & $\mathbf{2 3 , 4 8} \mathrm{ab}(5,38)$ & $\mathbf{1 4 , 9 4} \mathrm{ab}(7,90)$ \\
$\mathbf{4 , 0} \times \mathbf{4 , 0}$ & $\mathbf{2 3 , 1 3} \mathrm{c}(9,03)$ & $\mathbf{2 3 , 8 7} \mathrm{ab}(8,41)$ & $\mathbf{1 3 , 2 3} \mathrm{b}(8,02)$ \\
\hline
\end{tabular}

${ }^{*}$ Médias seguidas de uma mesma letra na coluna não diferem significativamente pelo teste de Tukey ao nível de $5 \%$. ${ }^{*}$ Valores de coeficiente de variação (\%); DAP: Diâmetro na altura de 1,30 m do solo; Ht: Altura total da árvore; Hic: Altura da inserção da copa. 
usada para a modelagem do crescimento de árvores individuais (Pretzsch, 1995; Spathelf, 1999).

Os valores do coeficiente de variação foram baixos para as três variáveis de crescimento, independentemente do espaçamento, o que demonstra uma menor variabilidade entre os materiais, ou seja, tendem a um comportamento mais homogêneo.

Ao se analisar a relação entre o DAP e o tamanho da copa, observou-se uma ampla diversificação do diâmetro dos eucaliptos aos 5 anos de idade (Figura 3). O DAP variou entre 15 a $25 \mathrm{~cm}$ nas árvores dentro das parcelas experimentais, ao se incluir as cinco árvores mais grossas da bordadura, observam-se diâmetros até $35 \mathrm{~cm}$ na idade de 5 anos. Verificou-se que o DAP é diretamente correlacionado com o diâmetro da copa $(r=0,96)$, ou seja, quanto maior a área de copa, consequentemente, ocorre também o aumento do DAP.

Wink et al. (2012), ao estudarem plantios homogêneos de eucalipto, verificaram que o comprimento de copa, o diâmetro, a área de projeção para a percentagem de copa aumentaram com a idade da floresta e com o DAP das árvores.

O modelo linear simples foi: diâmetro copa $(\mathrm{m})=0,1911^{\star} \operatorname{DAP}(\mathrm{cm})-1,2255$, com um coeficiente de determinação de 0,92, indica que um espaço vital maior para a árvore individual resulta em um melhor crescimento em diâmetro. Esse fato foi comprovado para outras espécies de eucalipto (Biechele et al., 2009; Nutto et al., 2006; Medhurst et al., 1999). Os modelos, abrangendo diferentes classes de idade e condições

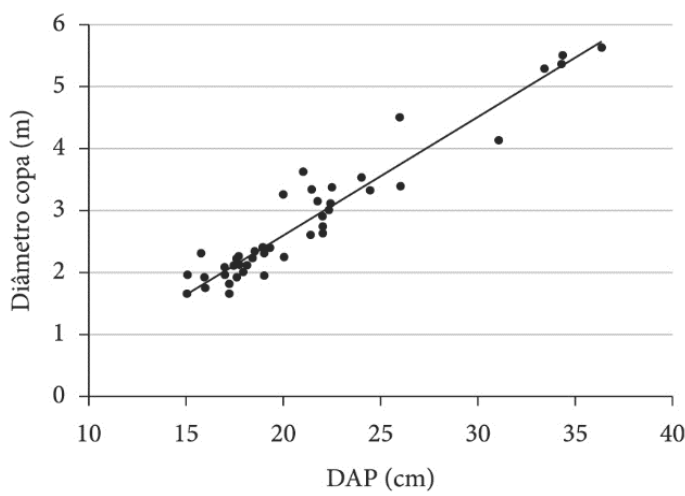

Figura 3. Comportamento do diâmetro da copa em função do diâmetro à altura de $1,30 \mathrm{~m}$ do solo para Eucalyptus benthamii aos 5 anos de idade.

Figure 3. Behavior of crown diameter to the diameter height of $1.30 \mathrm{~m}$ for Eucalyptus benthamii to 5 years old. de crescimento, podem ser usados para determinar a melhor idade e a frequência de desbastes para se atingir um determinado crescimento em diâmetro, conduzindo-se o manejo pela área de copa das árvores.

Os valores médios DRL obtidos nas direções norte, leste, sul e oeste para os diferentes espaçamentos estão apresentados na Tabela 2. Os valores encontrados estão muito acima quando comparados com outras espécies do gênero Eucalyptus. Muneri et al. (2000), para a espécie E. cloeziana com 4 anos de idade $(0,077 \mathrm{~mm})$, Lima et al. (2004) em clones de Eucalyptus spp. com idade variando de 8,5 a 15 anos (0,071 mm), Trugilho (2005) para 11 clones de Eucalyptus spp. com 6 anos de idade (0,090 mm), Cardoso et al. (2005) para clones de Eucalyptus com 5 anos de idade (0,065 mm), Rodrigues et al. (2008) para 13 clones de Eucalyptus spp. com 10 anos de idade (0,081 mm) e Beltrame et al. (2012) para Eucalyptus spp. com 9 anos de idade $(0,111 \mathrm{~mm})$.

Ao se comparar os diferentes espaçamentos, observou-se que o espaçamento 2,0 × 3,0 m apresentou os menores valores médios para DRL, diferindo apenas do espaçamento $3,0 \times 3,0 \mathrm{~m}$, indicando que o aumento do espaçamento não alterou significativamente os valores da DRL. Supõe-se que, provavelmente, menores áreas úteis da parcela geraram para o E. benthami menores níveis de tensão de crescimento.

Verificou-se que os valores médios da DRL nas quatro posições cardeais foram variáveis. Estatisticamente não houve diferença significativa entre os pontos cardeais norte, leste e oeste, no entanto os menores valores médios da DRL foram observados na direção sul, indicando menores níveis de tensão de crescimento. Os diferentes valores encontrados provavelmente podem estar associados às condições ambientais e fatores internos de constituição do tronco. De acordo com Rodrigues et al. (2008), a diferença da DRL nos Eucalyptus pode estar associada às variações na madeira, em função da formação da madeira de tração. Burguer \& Richter (1991) ressaltam que fenômenos como insolação e crescimento desequilibrado das copas podem resultar na formação de madeira com propriedades desiguais.

Com relação à DRL (Tabela 2 ), verificou-se a variabilidade dos valores de coeficiente de variação nos diferentes espaçamentos, servindo como variável nas decisões quanto aos tratos silviculturais e manejos adotados. Para os quatro diferentes espaçamentos não 
Tabela 2. Valores médios e coeficiente de variação da deformação residual longitudinal para os diferentes espaçamentos em plantios de Eucalyptus benthamii aos 5 anos de idade.

Table 2. Mean values and coefficient of variation of the longitudinal residual strain for different spacings Eucalyptus benthamii of a 5 years old.

\begin{tabular}{|c|c|c|c|c|c|}
\hline \multirow{2}{*}{$\begin{array}{l}\text { Espaçamento } \\
(\mathbf{m} \times \mathbf{m})\end{array}$} & \multicolumn{4}{|c|}{ Deformação residual longitudinal (mm) } & \multirow[b]{2}{*}{ Média } \\
\hline & Norte & Leste & Sul & Oeste & \\
\hline $2,0 \times 3,0$ & $\begin{array}{c}\mathbf{0 , 1 2 8} \\
(43,79)^{\star *}\end{array}$ & $\begin{array}{r}\mathbf{0 , 1 4 4} \\
(23,02)\end{array}$ & $\begin{array}{r}\mathbf{0 , 1 1 2} \\
(23,74)\end{array}$ & $\begin{array}{r}\mathbf{0 , 1 2 9} \\
(28,82)\end{array}$ & $0,128 b^{\star}$ \\
\hline $3,0 \times 3,0$ & $\begin{array}{c}\mathbf{0 , 1 5 4} \\
(20,86)\end{array}$ & $\begin{array}{r}\mathbf{0 , 1 6 7} \\
(29,57)\end{array}$ & $\begin{array}{r}\mathbf{0 , 1 2 6} \\
(36,58)\end{array}$ & $\begin{array}{r}\mathbf{0 , 1 4 0} \\
(13,24)\end{array}$ & 0,147 a \\
\hline $3,0 \times 4,0$ & $\begin{array}{r}\mathbf{0 , 1 5 5} \\
(31,20)\end{array}$ & $\begin{array}{r}\mathbf{0 , 1 5 2} \\
(14,24)\end{array}$ & $\begin{array}{r}\mathbf{0 , 1 3 3} \\
(35,53)\end{array}$ & $\begin{array}{r}\mathbf{0 , 1 3 7} \\
(19,05)\end{array}$ & $0,144 \mathrm{ab}$ \\
\hline $4,0 \times 4,0$ & $\begin{array}{c}\mathbf{0 , 1 7 4} \\
(22,72)\end{array}$ & $\begin{array}{r}\mathbf{0 , 1 3 5} \\
(42,47)\end{array}$ & $\begin{array}{r}\mathbf{0 , 1 1 9} \\
(42,16)\end{array}$ & $\begin{array}{r}\mathbf{0 , 1 4 4} \\
(34,49)\end{array}$ & $0,143 \mathrm{ab}$ \\
\hline Média & $0,153 A^{*}$ & $0,150 \mathrm{~A}$ & $0,123 \mathrm{~B}$ & $0,138 \mathrm{AB}$ & 0,141 \\
\hline
\end{tabular}

*Médias seguidas de uma mesma letra maiúscula na linha e minúscula na coluna não diferem significativamente pelo teste de Tukey ao nível de $5 \%$. ${ }^{* *}$ Valores de coeficiente de variação (\%).

Tabela 3. Análise de correlação entre os valores médios e máximo da DRL com as variáveis de crescimento para o Eucalyptus benhtamii aos 5 anos de idade.

Table 3. Analysis of correlation between the average and maximum DRL with the growth variables for Eucalyptus benthamii of a 5 years old.

\begin{tabular}{clllll} 
DRL & DAP & Ht & Ht/DAP & Dc & Exc \\
\hline Médio & $0,06386 \mathrm{~ns}$ & $0,26664 \mathrm{~ns}$ & $0,01124 \mathrm{~ns}$ & $0,06559 \mathrm{~ns}$ & $0,05542 \mathrm{~ns}$ \\
Máximo & $0,36841^{*}$ & $0,13027 \mathrm{~ns}$ & $-0,2774^{*}$ & $0,36206^{* *}$ & $0,44002^{* * *}$ \\
\hline
\end{tabular}

ns $=$ não significativo. ${ }^{*}=10 \% .{ }^{* *}=5 \% .{ }^{* *}=1 \%$ de probabilidade. DAP: Diâmetro na altura do peito; Ht: Altura total da árvore; Dc: Diâmetro da copa; Exc: Excentricidade da copa.

foi verificada qualquer tendência de comportamento característico quando se relacionaram DAP e DRL.

As médias da DRL dos diferentes espaçamentos mostram claramente o problema que ocorre quando trabalhamos com os valores médios dos povoamentos. Até esse ponto foi desconsiderado o efeito de falhas no plantio, quedas das árvores provocadas pelos ventos e a competição entre os diferentes indivíduos do experimento oriundos de semente que, com sua variabilidade genética, levam a uma autodiferenciação, mesmo disponibilizando-se um espaço vital idêntico para as árvores no plantio.

Ao se comparar os resultados obtidos com uma análise baseada no crescimento da árvore individual, com todos os indivíduos na idade de 5 anos na mesma análise, pôde-se observar mudanças no quadro. Enquanto os valores médios da DRL não apresentaram nenhuma correlação significativa com as variáveis de crescimento, nos valores máximos da DRL observou-se o contrário: alguns indicadores do crescimento foram correlacionados com as tensões máximas encontradas em cada indivíduo.
Considerando-se os valores médios e máximos da DRL, verifica-se na Tabela 3 que o maior coeficiente de correlação com uma alta probabilidade de significância foi encontrado na excentricidade da copa, seguido pelo diâmetro da copa, o DAP e o quociente entre altura e diâmetro. Resultados parecidos foram encontrados por Biechele et al. (2009) e Nutto \& Touza Vázquez (2006a). Os autores, porém, mostraram que a influência das variáveis de crescimento na DRL são dinâmicas, ou seja, mudam com a idade e a altura no fuste onde são medidas.

Silva (2008) também obteve baixa correlação entre as variáveis crescimento e níveis de tensão de crescimento. Segundo a autora, essa correlação negativa $(-0,45)$ favorece a seleção para ambas as variáveis estudadas.

\section{CONCLUSÕES}

Os plantios florestais de Eucalyptus benthamii apresentaram uma grande variabilidade das variáveis de crescimento e dos níveis de tensão de crescimento para os diferentes espaçamentos. 
As diferenças entre os espaçamentos foram parcialmente ocultadas pelo processo de autodiferenciação das árvores oriundas de semente. Isso pôde ser confirmado com a correlação forte entre o crescimento em diâmetro e o tamanho da copa, o que permitiu o desenvolvimento de um modelo de crescimento baseado na árvore individual.

Os valores da deformação residual longitudinal foram altos, mostrando-se superiores quando comparados às outras espécies do mesmo gênero. Os menores valores médios de DRL foram observados na direção sul. Foi observado que o aumento do espaçamento não alterou significativamente os valores da DRL.

Uma correlação significativa entre as variáveis de crescimento e a deformação residual longitudinal somente existiu para o valor máximo da DRL.

Recomenda-se a avaliação do nível de tensão juntamente com as variáveis de crescimento em idades superiores em diferentes espaçamentos para avaliação da tendência de comportamento ao correlacionarem-se as variáveis em estudo.

\section{STATUS DA SUBMISSÃO}

Recebido: 23 maio, 2013

Aceito: 17 fev., 2015

\section{AUTOR(ES) PARA CORRESPONDÊNCIA}

\section{Graziela Baptista Vidaurre}

Departamento de Ciências Florestais e da Madeira, Universidade Federal do Espirito Santo, Av. Governador Lindemberg, 316, Centro, Jeronimo Monteiro, ES, Brasil e-mail: grazividaurre@gmail.com

\section{REFERENNCIAS}

Alzate SBA, Tomazello M Fo, Piedade SMS. Variação longitudinal da densidade básica da madeira de clones de Eucalyptus grandis Hill ex Maiden, Eucalyptus grandis Hill ex Maiden, E. saligna Sm. e E. grandis x E. urophylla. Scientia Forestalis 2005; 68: 87-95.

Archer RR. Growth stresses and strains in trees. Berlin: Springer-Verlag; 1986.

Archer RR. On the origin of growth stresses in trees. Wood Science and Technology 1987; 21(2): 139-154. http:// dx.doi.org/10.1007/BF00376194.
Beltrame R, Lazarotto M, Haselein CR, Santini EJ, Schneider PR, Aguiar AM. Determinação das deformações residuais longitudinais decorrentes das tensões de crescimento em Eucalyptus spp. Ciência Florestal 2012; 22(2): 343-351. http://dx.doi.org/10.5902/198050985741.

Biechele T, Nutto L, Becker G. Growth strain in Eucalyptus nitens at different stages of development. Silva Fennica 2009; 43(4): 669-679. http://dx.doi.org/10.14214/sf.187.

Burguer ML, Richter HG. Anatomia da madeira. São Paulo: Nobel; 1991.

Cardoso A Jr. Bracatinga. Brasil Madeira 1989; 3(33): 1-10.

Cardoso AA Jr, Trugilho PF, Lima JL, Rosado SCS, Mendes LM. Deformação residual longitudinal em diferentes espaçamentos e idades em clone de híbrido de Eucalyptus. Cerne 2005; 11(3): 218-224.

Cardoso AA Jr, Trugilho PF, Lima JL, Rosado SCS. Tratos silviculturais na deformação residual longitudinal em clones de eucalipto. Scientia Forestalis 2007; 75: 77-84.

Evert F. Spacing studies: a review. Ottawa: Forest Management Institute; 1971. 95 p. Information Report FMR X 37.

Ferrand JC. Growth stresses and silviculture of eucalyptus. Australian Forest Research 1983; 13(1): 75-81.

Godoy H, Correa AR, Santos D. Clima do Paraná. In: Instituto Agronômico do Paraná - IAPAR. Manual agropecuário para o Paraná. Londrina; 1976.

Kubler H. Growth stresses in trees and related wood properties. Forestry-Abstracts 1987; 48: 131-189.

Kubler H. Silvicultural control of mechanical stresses in trees. Canadian Journal of Forest 1988; 18(10): 1215-1225. http://dx.doi.org/10.1139/x88-188.

Lemos F. Wachstumsspannungen in Eucalyptus spp: wechselwirkungen zwischen zuwachsrate, kronenasymmetrie und spannungsverteilung an der stammoberfläche [dissertação]. Freiburg: University of Freiburg; 2002.

Lima JT, Trugilho PF, Rosado SCZ, Cruz CR. Deformações residuais longitudinais decorrentes de tensões de crescimento em Eucalyptus e sua associação com outras propriedades. Árvore 2004; 28(1): 107-116.

Lisboa CDJ. Estudo das tensões de crescimento em toras de Eucalyptus grandis Hill ex Maiden [tese]. Curitiba: Ciências Florestais, Universidade Federal do Paraná; 1993.

Medhurst JL, Battaglia M, Cherry MLHMA, White DA, Beadle CL. Allometric relationships for Eucalyptus nitens (Deane and Maiden) Maiden plantations. Trees: Structure and Functions 1999; 14: 91-101.

Muneri A, Knigth J, Legate W, Palmer G. Relationships between surface longitudinal growth strain and tree size, wood properties and timber distorcion of 4 years old plantation grown Eucalyptus cloeziana. In: Proceedings of the Iufro Conference. The Future of Eucalyptus for Wood Products; 2000; Lauceston. Austrália. Launceston: IUFRO; 2000. p. 292-300. 
Nutto L, Spathelf P, Seling I. Management of individual tree diameter growth and implications for pruning brazilian Eucalyptus grandis Hill ex. Maiden. Floresta 2006; 36(3): 397-413.

Nutto L, Spiecker H. Production of valuable wood: A software aided decision tool for managing oak stands (Quercus petraea Liebl. and Q. robur L.) in Europe. Annales Experimentis Silvarum Culturae Provehendis 2000; 37: 37-49.

Nutto L, Touza Vázquez M. A new approach to model growth stresses in Eucalypts at different stages of development: do stress influencing parameters change during a trees life. In: Iufro Fifth Workshop, Connection between Forest Resources and Wood Quality: Modelling Approaches and Simulation Software; 2005; Auckland. New Zealand; $2006 a$.

Nutto L, Touza Vázquez MC. Modelos de producción de madera sólida en plantaciones de Eucalyptus globulus de Galicia. Boletin del CIDEU 2006b; 2: 37-50.

Pretzsch H. Perspektiven einer modellorientierten Waldwachstums-forschung. Forstwissenschaftliches Centralblatt 1995; 114(1): 188-209. http://dx.doi. org/10.1007/BF02742224.

Rodrigues EAC, Rosado SCS, Trugilho PF. Santos AMl. Seleção de clones de Eucalyptus para as propriedades físicas da madeira avaliadas em árvores no campo. Cerne 2008; 14(2): 147-152.

Röhle H. Vergleichende Untersuchungen zur Ermittlung der Genauigkeit bei der Ablotung von Kronenradien. Forstarchiv 1986; 57: 67-71.

Silva LD. Melhoramento genético de Eucalyptus benthamii Maiden et Cambage visando a produção de madeira serrada em áreas de ocorrencia de geadas severas [tese]. Curitiba: Setor de Ciências Agrárias, Universidade Federal do Paraná; 2008.

Spathelf P. Orientierungshilfe zur Prognose und Steuerung des Wachstums von Fichten (Picea abies (L.) Karst.) und Tannen (Abies alba Mill.) in Überführungswäldern mit Hilfe der relativen Kronenlänge [tese]. Freiburg: Universidade de Freiburg; 1999

Spiecker H. Orientierungshilfe für die Steuerung des Dickenwachstums vonEichen-Z-äumen. Allgemeine Forstzeitschrift 1983; 22: 569-570.

Trugilho PF. Tensão de crescimento em árvores vivas de clones de Eucalyptus spp. e de Eucalyptus dunnii Maiden e propriedades da sua madeira [tese]. Curitiba: Setor de Ciências Agrárias, Universidade Federal do Paraná; 2005.

Vidaurre GB, Lombardi LR, Nutto L, França FJN, Oliveira JTS, Arantes MDC. Propriedades da madeira de reação. Floresta e Ambiente 2013; 20(1): 26-37. http://dx.doi. org/10.4322/floram.2012.041.

Vignote S, Molinero I, Gerard J, Diez MR. Estudios de las tensiones de crecimiento del Eucalyptus globulus Labill. en Galicia y su relacion con las caracteristicas de la estacion y morfologicas del propio arbol. Investigacíon Agraria: Sistemas e Recursos Forestales 1996; 5(1): 153-176.

Wink C, Monteiro JS, Reinert DJ, Liberalesso E. Parâmetros da copa e a sua relação com o diâmetro e altura das árvores de eucalipto em diferentes idades. Scientia Florestalis 2012; 40(93): 57-67.

Yang JL, Waugh G. Growth stress, it's measurement and effects. Australian Forestry 2001; 64(2): 127-135. http:// dx.doi.org/10.1080/00049158.2001.10676176. 